Research article

\title{
Simultaneous odour-face presentation strengthens hedonic evaluations and event-related potential responses influenced by unpleasant odour
}

\author{
Stephanie Cook $^{\mathrm{a}, *}$, Katerina Kokmotou ${ }^{\mathrm{a}, \mathrm{b}}$, Vicente Soto ${ }^{\mathrm{a}}$, Hazel Wright ${ }^{\mathrm{a}}$, Nicholas Fallon ${ }^{\mathrm{a}}$, \\ Anna Thomas ${ }^{\mathrm{c}}$, Timo Giesbrecht ${ }^{\mathrm{c}}$, Matt Field ${ }^{\mathrm{a}}$, Andrej Stancak ${ }^{\mathrm{a}, \mathrm{b}}$

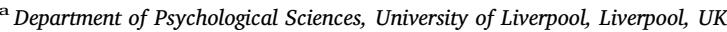 \\ ${ }^{\mathrm{b}}$ Institute for Risk and Uncertainty, University of Liverpool, Liverpool, UK \\ ${ }^{\mathrm{c}}$ Department of Research and Development, Unilever, Port Sunlight, UK
}

\section{A R T I C L E I N F O}

\section{Keywords:}

Olfaction

EEG

Perception

Time

\begin{abstract}
A B S T R A C T
Odours alter evaluations of concurrently presented visual stimuli, such as faces. Stimulus onset asynchrony (SOA) is known to affect evaluative priming in various sensory modalities. However, effects of SOA on odour priming of visual stimuli are not known. The present study aimed to analyse whether subjective and cortical activation changes during odour priming would vary as a function of SOA between odours and faces.

Twenty-eight participants rated faces under pleasant, unpleasant, and no-odour conditions using visual analogue scales. In half of trials, faces appeared one-second after odour offset (SOA 1). In the other half of trials, faces appeared during the odour pulse (SOA 2). EEG was recorded continuously using a 128-channel system, and event-related potentials (ERPs) to face stimuli were evaluated using statistical parametric mapping (SPM).

Faces presented during unpleasant-odour stimulation were rated significantly less pleasant than the same faces presented one-second after offset of the unpleasant odour. Scalp-time clusters in the late-positive-potential (LPP) time-range showed an interaction between odour and SOA effects, whereby activation was stronger for faces presented simultaneously with the unpleasant odour, compared to the same faces presented after odour offset.

Our results highlight stronger unpleasant odour priming with simultaneous, compared to delayed, odour-face presentation. Such effects were represented in both behavioural and neural data. A greater cortical and subjective response during simultaneous presentation of faces and unpleasant odour may have an adaptive role, allowing for a prompt and focused behavioural reaction to a concurrent stimulus if an aversive odour would signal danger, or unwanted social interaction.
\end{abstract}

\section{Introduction}

It is well known that olfaction and emotion are tightly linked [1], and that hedonic judgement is a key aspect of olfaction [2,3]. As a result, odours are able to evoke emotional states, and affect perceptual processes in other modalities [4]. Previous studies have shown that pleasant and unpleasant odours influence evaluations of human faces [5-12]. However, the neural mechanisms that underlie such effects are not well established. The few EEG studies investigating such effects revealed that late ERPs (such as the N400 and the late-positive potential, LPP) evoked by faces were modulated by the presence of pleasant and unpleasant odours [11,12]. Functional magnetic resonance imaging (fMRI) data suggested that faces paired with pleasant fragrance activated the medial orbitofrontal cortex implicated in encoding the reward value of stimuli; whilst faces paired with unpleasant odour activated the amygdala, known to be involved in the processing of aversive stimuli [9]. Such changes in hedonic evaluations of visual stimuli and associated brain activation patterns are described as odour priming effects [13].

Whilst the phenomenon of evaluative priming is well established in vision and semantics (reviewed in [14]), little is known about the specific, temporal aspects of odour priming effects [13]. Studies investigating affective priming using words and pictures suggest that the temporal association between primes and targets, known as stimulus onset asynchrony (SOA) is of importance $[15,16]$. A recent meta-analysis of evaluative priming pointed to SOA as a factor influencing the

\footnotetext{
* Corresponding author.

E-mail addresses: s.cook2@liverpool.ac.uk, stephanie.cook@ucl.ac.uk (S. Cook), a.kokmotou@liverpool.ac.uk (K. Kokmotou), v.soto@liverpool.ac.uk (V. Soto), h.wright@liverpool.ac.uk (H. Wright),n.b.fallon@liverpool.ac.uk (N. Fallon), anna.thomas@unilever.com (A. Thomas), timo.giesbrecht@unilever.com (T. Giesbrecht), mfield@liverpool.ac.uk (M. Field), stancak@liverpool.ac.uk (A. Stancak).
} 
strength of hedonic priming across various stimulus modalities [14]. The authors showed that SOA effects manifest in stronger changes in hedonic evaluation of targets with short, compared to long intervals between prime and target. However, there are no data on effects of SOA for olfactory priming.

To fill this gap in the literature, we investigate effects of two stimulus onset asynchronies on evaluative priming involving odours and faces. Further, we explore for the first time the neural manifestation of SOA in odour priming. In a previous study, we demonstrated odourpriming of neutral faces with both pleasant and unpleasant odours, when faces were presented one-second after odour offset. We also showed that pleasant odours increased the amplitude of face ERPs during the mid-late positive component (around $600 \mathrm{~ms}$ after face onset), and that pleasant and unpleasant odours respectively increased the amplitude of face ERPs in the left and right hemispheres, during the ultra-late positive component (around $900 \mathrm{~ms}$ after face onset) [11]. We now speculate about how the temporal variation between odour and face presentation affects both behavioural odour priming and neural responses to faces.

It has been proposed that early visual potentials (until approximately $300 \mathrm{~ms}$ after stimulus-onset) reflect unconscious stimulus perception, whilst later ones reflect conscious and controlled processing [17]. Hence, the late ERP effects observed in our previous study [11] may represent changes in the overt evaluation of faces that are necessary for priming after odour offset. During simultaneous odour-face presentation, odour-related effects may occur in earlier face-processing components (such as the N170, or N400) and be more representative of unconscious changes in face perception. At present, it is not known whether there are differences in effects of odours on hedonic evaluations of faces, either behaviourally or reflected in ERPs, when faces are presented during odour stimulation compared to when they are presented after odour offset.

The aim of this study was to investigate a direct comparison between odour priming with simultaneous and delayed presentation of odours and faces. In line with previous findings of SOA effects on the strength of evaluative priming [14], we hypothesised that odour-induced changes in evaluations of faces, early ERP components (e.g. N170, N400) and late ERP components (e.g. the LPP) may be stronger when faces appeared during the odour pulse compared to when they were presented one second after odour offset.

\section{Methods}

\subsection{Participants}

A total of 29 (10 male) participants aged 18-31 years (23.6 \pm 3.8 , mean \pm standard deviation) took part in the experiment after responding to an advertisement. All but 4 subjects were right-handed. One participant withdrew from the experiment. EEG data from two participants were subsequently excluded due to excessive amounts of artifacts. Hence, behavioural data from 28 subjects, and EEG data from 26 (10 male) subjects were used in the analysis. People suffering from asthma or neurological disorders, particularly anosmia or epilepsy, were not permitted to take part in the study. Normal olfactory function was ascertained using the Sniffin'Sticks [18] test battery. Participants had to successfully identify a minimum of 9 out of the 12 odours in order to take part in the experiment. Participants were asked not to smoke, drink coffee or chew gum for two hours prior to the experiment, and were asked to minimise their use of fragranced products on the day. Participants were reimbursed for their time and travel expenses. The study was approved by the Research Ethics Committee at the University of Liverpool. All participants gave written informed consent in accordance with the Declaration of Helsinki.

\subsection{Visual and olfactory stimuli}

A total of 90 (45 male) neutral faces were used in the experiment. Due to the large number of faces needed to satisfy the number of trials required per condition, faces were selected from three databases. Fortytwo (24 male) faces were obtained from the NimStim Set of Facial Expressions [19]. Forty-three (21 male) faces were obtained from the Japanese and Caucasian Neutral Faces [JACNeuf; [20]. A further five female faces were selected from the Gur/Kohler images, acquired according to Gur et al. [21] and referenced in Kohler et al. [22]. All face images were frontal views, in colour, with a consistent light background and similar dimensions. During the screening session, participants rated the perceived pleasantness of the facial expressions of all 90 faces (on a scale ranging from 0 -very unpleasant to 100 -very pleasant) in order to ensure that they were perceived as neutral. The mean face pleasantness rating was $50.3( \pm 8.4)$.

There were three odour conditions in the experiment; pleasant, unpleasant and a neutral, 'clean air' control. Methylmercaptan (1\% dilution in Propylene Glycol), a rotten cabbage-like odour, was selected for the unpleasant condition. Jasmine odour (no dilution) was selected for the pleasant condition. These dilutions were matched on perceived intensity based on data from a previous experiment [Mean intensity rating of Jasmine: $56.33 \pm 15.83$, mean intensity rating of Methylmercaptan: $61.34 \pm 17.68 ; 11]$.

\subsection{Procedure}

Procedures for odour administration, presentation of the experimental task, recording EEG and baseline odour ratings were identical to those described in previous papers $[11,23]$.

The experimental task was split into four blocks of 45 trials (180 trials in total). Trials were pseudo-randomly ordered such that each of the 90 faces used in the task appeared twice: once under each SOA condition, with the same odour both times. The odour with which a given face was paired was counterbalanced across subjects. Any given face never appeared more than once in one block. Odour presentation was also pseudo-random, such that all three odours were presented across all four blocks, but no two consecutive trials used the same odour. Fig. 1 shows a flowchart of the trial procedure. Each trial began with a resting interval during which participants viewed a white cross on a black background. The duration of this interval was dependent upon the triggering of the odour pulse; the experimenter observed participants' respiratory waveforms, and manually triggered the odour pulses at the very onset of inspiration. In half of the trials, a threesecond odour pulse was released, during which time participants viewed a black screen. The screen remained black for a further onesecond resting interval after odour offset, before a neutral face was displayed on-screen for $300 \mathrm{~ms}$ (SOA 1). The other half of the trials were identical, apart from that the neutral face was displayed on-screen during the three-second odour pulse, at $2000 \mathrm{~ms}$ after odour onset (SOA 2). In both conditions, a resting interval with a black screen then preceded a rating scale prompting participants to rate the pleasantness of the neutral face (from 0 -very unpleasant to 100 -very pleasant). Once participants had responded, a second scale prompted them to rate the intensity of the odour administered in that trial (0-no odour to 100 -very intense odour). After their response, the next trial began.

\subsection{Analysis}

Odour ratings taken before and after the task were analysed using paired $t$-tests. Data from the experimental task was analysed using $2 \times 3$ repeated measures ANOVA to observe differences in face pleasantness ratings and odour intensity ratings, with SOA and odour condition (pleasant, unpleasant, and neutral) as independent factors. All behavioural data were analysed using SPSS v. 22 (IBM Inc., USA). 


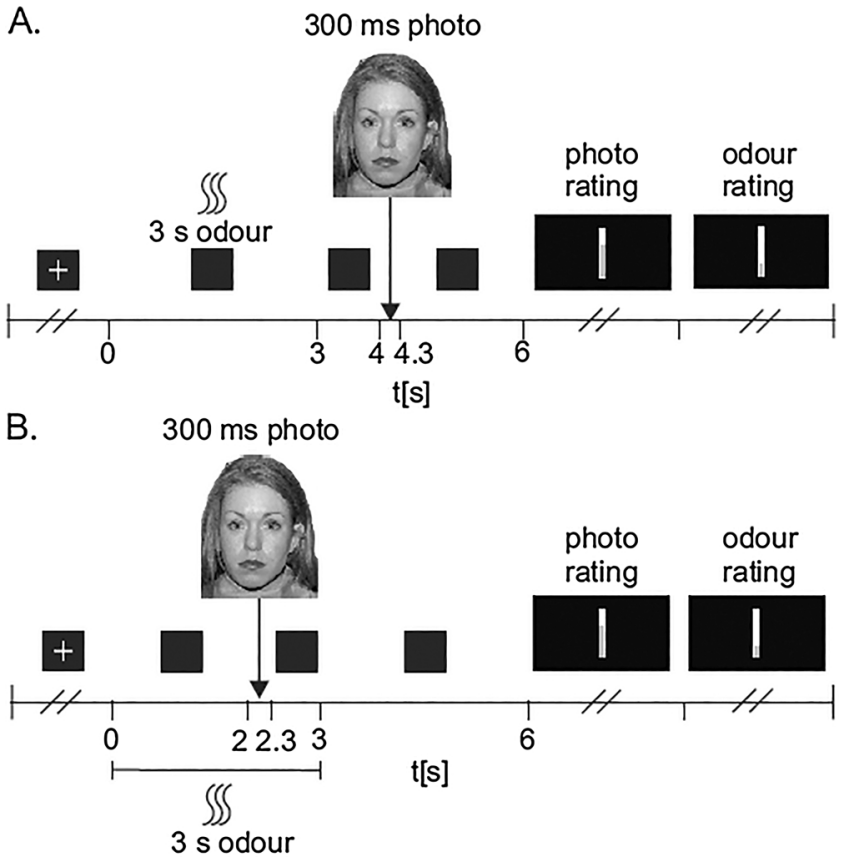

Fig. 1. Flowchart of experimental trial procedure. At the start of each trial, participants viewed a white fixation cross on a black background. Participants were instructed to relax and breathe normally during this time. At the very onset of a participant's inspiration, the experimenter triggered a three-second odour pulse. In half the trials (A), the odour pulse was followed by a one-second pause where participants viewed a black screen. Following this, a photograph of a neutral face was displayed for $300 \mathrm{~ms}$, followed by a $1700 \mathrm{~ms}$ rest period where participants viewed a black screen. In the other half of the trials (B), facephotographs appeared at $2000 \mathrm{~ms}$ during the odour pulse, for $300 \mathrm{~ms}$. In both types of trials, a visual-analogue scale prompted participants to rate the pleasantness of the photograph (very unpleasant - very pleasant), $6 \mathrm{~s}$ after odour onset. A second scale then prompted participants to rate the intensity of the odour (no odour - very intense odour). Once participants had completed both ratings, the next trial began.

\subsection{ERP analysis}

Pre-processing of EEG recordings followed the same procedure as described in our previous papers [11,23]. The mean number of accepted trials across all subjects and all conditions was $160(\mathrm{SD}=17.5)$. Participants were excluded from the analysis if the number of trials accepted was less than 125 ( 2 standard deviations from the mean). The mean numbers of accepted trials for each condition were as follows: Clean air SOA 1: 28, Clean air SOA 2: 29, Jasmine SOA 1: 29, Jasmine SOA 2: 28, Methylmercaptan SOA 1: 28, Methylmercaptan SOA 2: 27.

EEG data filtering, sampling, epoching and analysis was carried out using the same procedure as described in previous work [23]. Eventrelated potentials (ERPs) in response to neutral faces were analysed using a $2 \times 3$ flexible factorial model in SPM12 (Statistical Parametric Mapping, UCL, England; http://www.fil.ion.ucl.ac.uk/spm/software/ spm12/), where SOA and odour condition were the independent variables. We therefore examined the effects of odour and SOA on EEG data across all electrodes and time points from 0 to $1 \mathrm{~s}$ relative to the onset of the face images, in order to achieve robust and unbiased results as opposed to analysing specific pre-selected ERP components. An uncorrected threshold of $\mathrm{P}<0.001, \mathrm{k}=20$ was applied to detect scalptime clusters significantly affected by odours and SOA. Amplitude data from significant clusters were analysed using further repeated measures ANOVAs in SPSS, with a statistical threshold of $\mathrm{P}<0.05$.

\section{Results}

\subsection{Odour ratings}

Mean ratings of odour pleasantness, intensity and familiarity taken
Table 1

Mean ( \pm standard deviation) ratings of odour pleasantness, intensity and familiarity that were taken before and after the experimental task and concatenated.

\begin{tabular}{llll}
\hline Odour & Pleasantness & Intensity & Familiarity \\
\hline Jasmine & $78.17( \pm 11.15)$ & $75.56( \pm 10.86)$ & $68.36( \pm 20.53)$ \\
Methylmercaptan & $13.0( \pm 10.38)$ & $80.09( \pm 9.69)$ & $65.71( \pm 24.45)$ \\
\hline
\end{tabular}

Table 2

Mean ( \pm standard deviation) pleasantness ratings of neutral faces under each odour and SOA condition (SOA 1-faces presented one second after odour offset, SOA 2-faces presented during odour stimulation).

\begin{tabular}{llll}
\hline SOA & Clean air & Jasmine & Methylmercaptan \\
\hline SOA 1 & $53.76( \pm 6.23)$ & $53.30( \pm 7.57)$ & $49.39( \pm 5.75)$ \\
SOA 2 & $54.11( \pm 7.10)$ & $53.81( \pm 7.81)$ & $47.94( \pm 7.58)$ \\
\hline
\end{tabular}

before and after the experimental task were collated and are shown in Table 1. Paired $t$-tests confirmed that jasmine was rated as significantly more pleasant than methylmercaptan $(\mathrm{t}(27)=28.34, \mathrm{P}<0.001)$; there was no significant difference in intensity ratings of jasmine and methylmercaptan $(\mathrm{t}(27)=-1.64, \mathrm{P}=0.11)$, and there was no significant difference in familiarity ratings of jasmine and methylmercaptan $(\mathrm{t}(27)=4, \mathrm{P}=0.48)$.

\subsection{Face ratings}

Table 2 shows the mean ratings of faces under each odour and SOA condition. A repeated-measures ANOVA revealed a significant main effect of odour on ratings of faces $\left(\mathrm{F}(2,54)=14.63, \eta_{p}^{2}=0.35\right.$, $\mathrm{P}<0.001$ ). Pairwise comparisons indicated that faces in the unpleasant odour condition were rated as significantly less pleasant in comparison to faces in both the control $(\mathrm{P}<0.001)$ and pleasant odour ( $\mathrm{P}=0.001)$ conditions. There was no significant difference between ratings of faces in the control and pleasant odour conditions $(\mathrm{P}>0.05)$. There was no main effect of SOA on ratings of faces $(\mathrm{F}(1$, $\left.27)=0.23, \eta_{p}^{2}=0.01, \mathrm{P}=0.64\right)$. However, there was a significant interaction between odours and SOA affecting face ratings $(\mathrm{F}(2$, $\left.54)=3.3 \eta_{p}^{2}=0.11, \mathrm{P}=0.05\right)$. Further analysis of this interaction (using a $2 \times 2$ repeated measures ANOVA) showed that the effect was caused by the greater pleasantness of faces presented during averaged clean air and pleasant odour conditions and greater unpleasantness of faces presented during the unpleasant odour condition in SOA2 compared to SOA1 $(\mathrm{F}(1,27)=5.02, \mathrm{P}=0.034)$. The interaction appeared to be driven by the contrast between SOAs in the unpleasant odour condition $(\mathrm{F}(1,27)=3.29, \mathrm{P}=0.081)$, since this contrast was comparatively weak in the pleasant odour and clean air conditions $(\mathrm{F}(1$, $27)=1.07, P=0.31$ ). Further one-way ANOVAs were computed to analyse the effect of odour in each SOA condition separately. These revealed an effect of odour in both SOA conditions (SOA 1: $(\mathrm{F}(2$, $\left.54)=8, \quad \eta_{p}^{2}=0.23 \quad \mathrm{P}=0.002\right) ; \quad \mathrm{SOA} \quad 2: \quad(\mathrm{F}(2, \quad 54)=18.94$, $\left.\eta_{p}^{2}=0.41 \mathrm{P}<0.001\right)$ ), where the effect of odour in SOA 1 was nonsignificant after Bonferroni correction for multiple comparisons $(P>0.025)$.

\subsection{ERP components}

Fig. 2 illustrates the event-related potentials in response to faces across all trials and all odour conditions in the form of a butterfly plot and topographic maps of selected potential components. Topography of the first component showed bilateral positivity over the occipital electrodes and negativity over frontal electrodes, peaking at around $100 \mathrm{~ms}$. This is consistent with characteristics of the P1 component, which is related to early processing of visual stimuli [24]. The second component, peaking around $205 \mathrm{~ms}$, showed negativity over parietal and 
A.

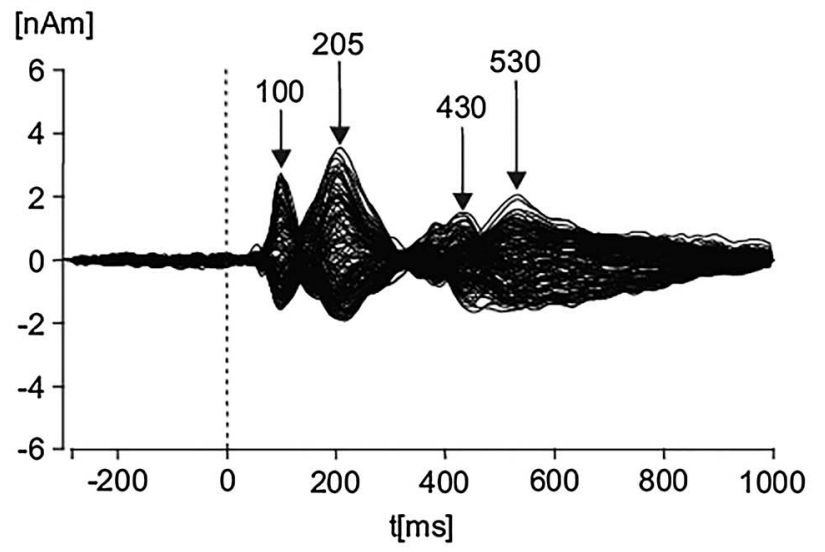

B.

B. $100 \mathrm{~ms}$
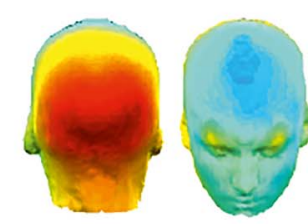

$[\mu \mathrm{V}]$

D.

$430 \mathrm{~ms}$

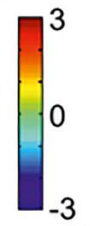

C. $205 \mathrm{~ms}$

$[\mu \mathrm{V}]$

E.
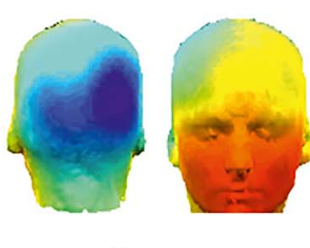

$[\mu \mathrm{V}]$
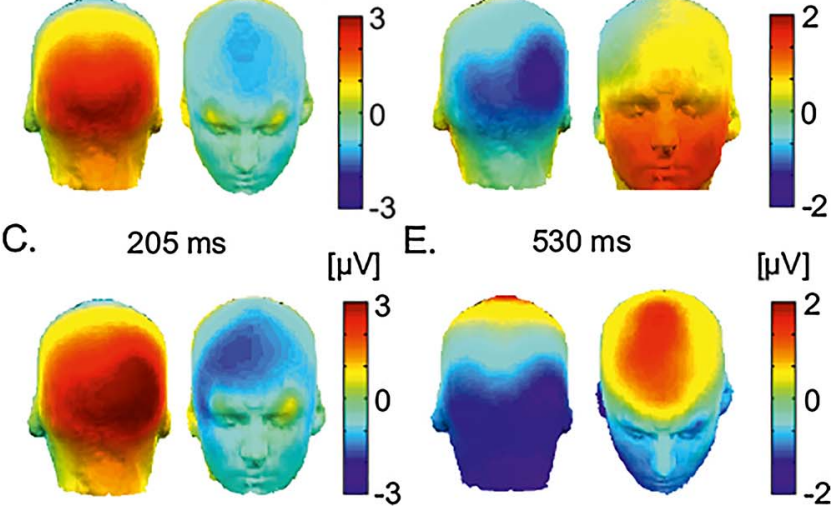

$530 \mathrm{~ms}$

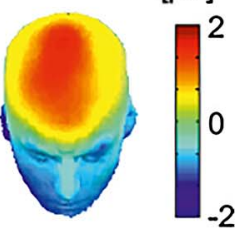

Fig. 2. Butterfly plot of grand average ERP response to faces and corresponding scalp topographies. (A) Butterfly plot of grand average ERPs in response to faces. Peak latencies of distinct ERP components $(100 \mathrm{~ms}, 205 \mathrm{~ms}, 430 \mathrm{~ms}$, and $530 \mathrm{~ms}$ ) are highlighted with arrows. (B) Latency component $100 \mathrm{~ms}$ (P1). The topographic maps of grand average ERPs overlaid on the volume rendering of the human head are shown. (C) Latency component $205 \mathrm{~ms}$ (N170). (D) Latency component $430 \mathrm{~ms}$ (N400). (E) Latency component $530 \mathrm{~ms}$ (late component/LPP).

temporal electrodes, consistent with characteristics of the N170 faceprocessing component [25]. The next component peaked around $430 \mathrm{~ms}$, showing strong negativity over occipital and parietal electrodes, consistent with the N400 component, which is implicated in the processing of meaningful stimuli, including faces [26]. The final component was a long component beginning around $500 \mathrm{~ms}$ and peaking at approximately $530 \mathrm{~ms}$. Showing negativity over occipital electrodes sites and positivity over central areas, it was consistent with characteristics of the late positive potential (LPP), which is sensitive to the emotional content of pictures, words and faces [27-30].

\subsection{Effects of odours and SOA on ERPS}

SPM1 2 was used to compute a $2 \times 3$ repeated measures ANOVA on smoothed scalp-time images of data from $0-1000 \mathrm{~ms}$ relative to the onset of the face. The ANOVA revealed scalp-time clusters showing significant interaction effects of SOA and odour on the ERP response to faces, defined by their peak latencies.

There was a main effect of SOA on ERP response to faces in clusters peaking at $169 \mathrm{~ms}$ and $173 \mathrm{~ms}$ after onset of the face (uncorrected $\mathrm{P}<0.001$ ), during the N170 time-window. Subsequent $t$-tests performed on EEG amplitude data from these two clusters showed that faces presented using SOA 2 yielded stronger EEG amplitude at both the 169 ms cluster $(\mathrm{t}(25)=-5.49, \mathrm{P}<0.001)$, and the $173 \mathrm{~ms}$ cluster $(\mathrm{t}$ $(25)=5.67, \mathrm{P}<0.001)$ compared to faces presented using SOA 1 .
An interaction between odour and SOA yielded a significant effect (unc. $\mathrm{P}<0.001$ ) on the ERP response to faces in scalp-time clusters peaking at $516 \mathrm{~ms}\left(\mathrm{~F}(2,50)=9.81 \eta_{p}^{2}=0.28, \mathrm{P}=0.001\right)$, and $712 \mathrm{~ms}$ $\left(\mathrm{F}(2,50)=12.81, \eta_{p}^{2}=0.34, \mathrm{P}<0.001\right)$ following onset of the face. Fig. 3 illustrates these significant scalp-time clusters. The corresponding topographic maps from each odour/SOA condition for each significant cluster are shown with bar graphs representing the mean EEG scalpamplitude $(\mu \mathrm{V})$. Post-hoc $t$-tests revealed significantly greater amplitudes of positive and negative potential components for faces presented in the unpleasant odour condition using SOA 2, in the $516 \mathrm{~ms}$ cluster $(1.00 \pm 0.60, \mathrm{P}=0.002)$ and the $712 \mathrm{~ms}$ cluster $(-0.90 \pm 0.73$, $\mathrm{P}<0.001$ ), respectively. There were no significant differences in EEG amplitude between SOA 1 and SOA 2 in the clean air or pleasant odour conditions $(\mathrm{P}>0.05)$.

\section{Discussion}

A direct comparison of concurrent versus delayed stimulation in odour priming of neutral faces revealed effects that manifested in hedonic evaluations and cortical potentials. The effects of odour on hedonic evaluations of faces were clearly stronger in the concurrent stimulation condition. In particular, unpleasant odours had a greater effect on both hedonic evaluations and cortical responses when faces were presented during odour stimulation.

In accordance with previous studies [5,8,11], neutral faces presented with or after unpleasant odour stimulation were rated as significantly less pleasant than faces in both the control and pleasant odour conditions. However, the present study specifically demonstrated that unpleasant odour priming effects occurred both with and without a temporal lag between odour and face presentation Moreover, as hypothesised, the influence of unpleasant odour on evaluations of faces was stronger during concurrent stimulation, consistent with work reporting stronger evaluative priming with short prime-target intervals [14].

The temporal association between odour and face presentation modulated early face-processing. SOA significantly affected scalp-time clusters in the N170 time-range, showing stronger activation when faces were presented during odour stimulation in comparison to when the same faces were presented one second after odour offset. This finding is consistent with the suggestion that odours may influence early stages of visual processing [31]. Indeed, one recent study showed enhancement of the EEG response between 130 and $180 \mathrm{~ms}$ after face onset when faces were presented with an odour [32]. It is likely that the multisensory stimulation experienced when odours and faces were presented together resulted in increased allocation of attentional resources and increased N170 amplitude as a consequence.

The key behavioural finding referred to an interaction between odour condition and SOA where faces presented during unpleasant odour stimulation were rated as less pleasant than the same faces presented one second after unpleasant odour offset. This interaction was mirrored in our EEG data during the late-positive potential (LPP) timerange ( $>500 \mathrm{~ms}$ ) [27]. Our data showed that faces presented during unpleasant odour stimulation were associated with greater LPP amplitude than the same faces presented one second after offset of the unpleasant odour. Moreover, faces presented during unpleasant odour stimulation produced stronger LPP amplitude than those presented during pleasant odour stimulation or in the clean air condition. The LPP is known to be sensitive to the valence of pictures, words and faces [27-30]. Previous research has suggested that the LPP responds to the emotional content of faces [33], and that contextual information integrates with face processing during the LPP [34]. The present results further suggest that the LPP may indeed be sensitive to the emotional content of faces, or likely the emotional context in which they are presented, and may reflect the overt evaluations of faces that result from pairings with contextual odours. More specifically, odour context 


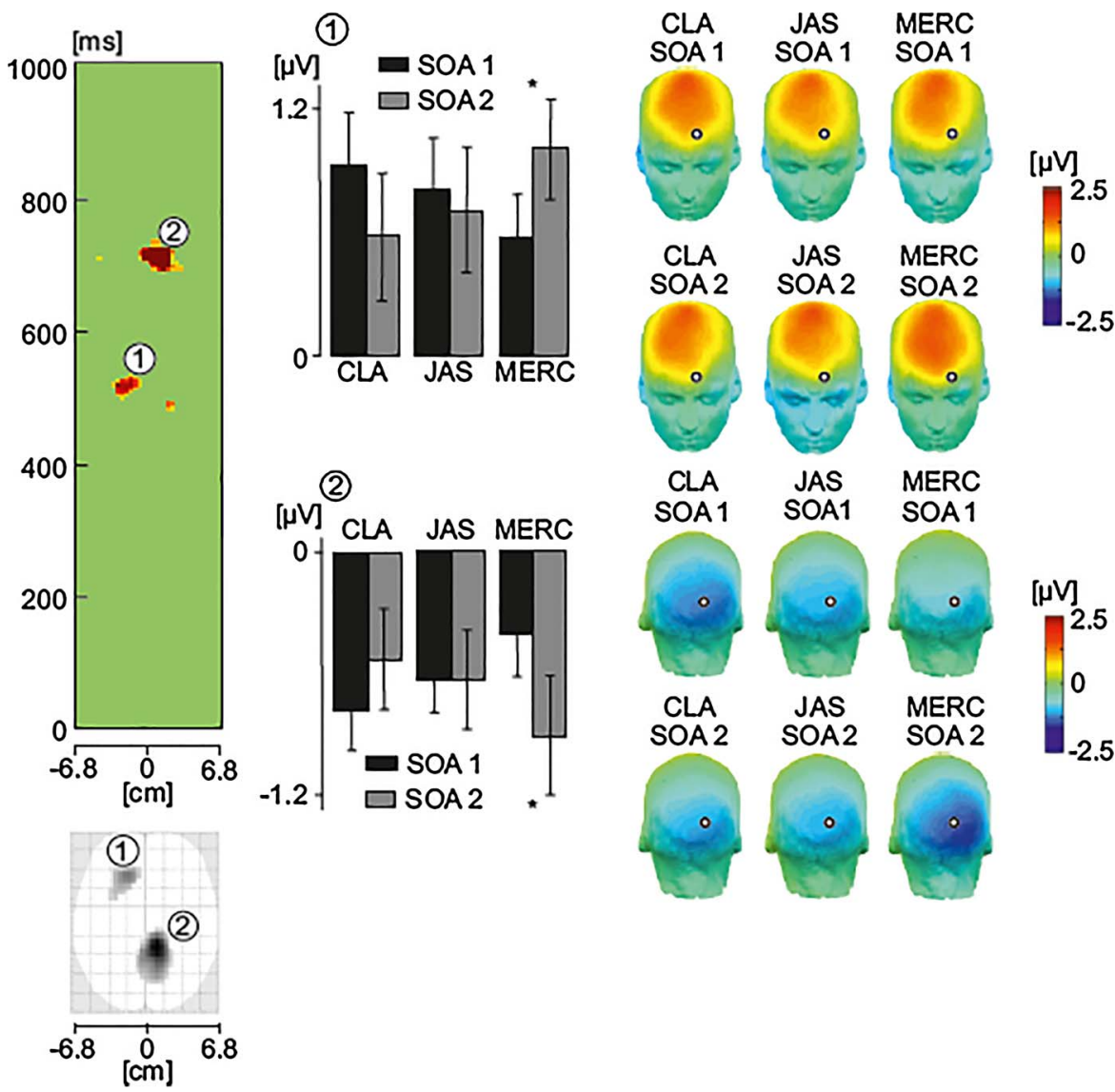

Fig. 3. Interaction between odour and SOA condition affecting ERP response to faces. The green panel shows statistically significant latency periods $(\mathrm{P}<0.001$ uncorrected) in the scalp-time plot. Two spatio-temporal clusters during the LPP were significantly affected by an interaction between odour and SOA conditions. Below the green panel is the standard scalp map of the statistically significant clusters. The first significant cluster, labelled 1, occurred at $516 \mathrm{~ms}$ and had a positive amplitude. The second, labelled 2, occurred at $712 \mathrm{~ms}$ and had a negative amplitude. Bar graphs illustrate the mean EEG amplitude for each cluster under each condition $(\mu \mathrm{V})$. Black bars represent SOA 1, and grey bars represent SOA 2. Odour conditions are labelled CLA, JAS, and MERC. Asterisks indicate statistically significant differences ( $\mathrm{P}<0.025$, Bonferroni corrected). Corresponding topographic maps of the numbered significant clusters for all conditions are shown. (For interpretation of the references to color in this figure legend, the reader is referred to the web version of this article.) interacted with SOA to influence face processing at the LPP.

Taken together, our results suggest that unpleasant odour priming effects were stronger during simultaneous odour and face presentation, and that the LPP may represent the strength of the effects of unpleasant odour context on face evaluation that occur as a result of the temporal association between odour and face. Stronger LPP amplitude and more significant changes in hedonic ratings during simultaneous unpleasant odour and neutral face stimulation support the evidence for an attentional bias towards negative stimuli [35-37], and are consistent with previous findings that negative odours elicit faster reactions than other odours [38]. A greater cortical and subjective response during simultaneous olfactory and visual stimulation may have an adaptive role, allowing for a prompt and focused behavioural reaction if an aversive odour would signal unwanted social interaction, or danger.

\section{Conclusion}

The aim of this study was to directly compare the effects of odours on hedonic evaluations of faces in the instance of simultaneous versus delayed odour-face presentations. The results suggest that unpleasant odour influences hedonic evaluations of faces with or without a temporal lag between the odour and the face. However, unpleasant odour priming is stronger using simultaneous odour-face presentation. Such an effect resulted in stronger negative evaluations of neutral faces, and stronger activation during the LPP time-window. Indeed, multiple senses work together to reveal more information about biologically meaningful events [39], and odours play a direct role in social interaction [5]. Unpleasant odours tend to repel us from such social interaction due to their adaptive signalling of danger, poisons, or infection. A stronger priming effect of unpleasant odours for concurrently occurring visual stimuli compared to stimuli occurring later may help to shape a more robust and focused behavioural response by tuning the hedonic evaluations and cortical responses to the visual stimulus towards the unpleasant pole. Such multimodal effects may allow for prompt mobilisation of behavioural resources to avoid unwanted social interaction, or tackle potential danger.

\section{Funding}

This work was supported by a CASE studentship from the Economic and Social Research Council (ESRC), with additional support from Unilever Research and Development [grant number: ES/J500094/1].

\section{Conflict of interest}

Authors Dr. A. Thomas and Dr. T. Giesbrecht are employed by Unilever. Unilever is interested in behavioural and cognitive effects of odours, as odours are natural ingredients of food and personal care products. However, none of the odours used in the present study are used commercially by Unilever. Unilever had minimal involvement in the study design; in the collection, analysis or interpretation of the data; in the writing of the report; and in the decision to submit the article for publication. The authors declare that the research was conducted in the absence of any commercial or financial relationships that could be construed as a potential conflict of interest.

\section{References}

[1] J.A. Gottfried, R. Deichmann, J.S. Winston, et al., Functional heterogeneity in human olfactory cortex: an event-related functional magnetic resonance imaging study, J. Neurosci. 22 (2002) 10819-10828.

[2] R.S. Herz, T. Engen, Odor memory: review and analysis, Psychon. Bull. Rev. 3 (1996) 300-313.

[3] M. Bensafi, C. Rouby, V. Farget, et al., Influence of affective and cognitive judgments on autonomic parameters during inhalation of pleasant and unpleasant odors in humans, Neurosci. Lett. 3 (2002) 162-166.

[4] H. Ehrlichman, J.N. Halpern, Affect and memory: effects of pleasant and unpleasant odors on retrieval of happy and unhappy memories, J. Pers. Soc. Psychol. 5 (1988) 
769-779.

[5] J. Todrank, D. Byrnes, A. Wrzesniewski, et al., Odors can change preferences for people in photographs - a cross-modal evaluative conditioning study with olfactory uss and visual css, Learn. Motiv. 26 (1995) 116-140.

[6] J.M. Leppanen, J.K. Hietanen, Affect and face perception odors modulate the recognition advantage of happy faces, Emotion 4 (2003) 315-326.

[7] M.L. Dematte, R. Osterbauer, C. Spence, Olfactory cues modulate facial attractiveness, Chem. Senses 32 (2007) 603-610.

[8] W. Li, I. Moallem, K.A. Paller, et al., Subliminal smells can guide social preferences, Psychol. Sci. 18 (2007) 1044-1049.

[9] F. McGlone, L.M. Demattè, C. Spence, The crossmodal influence of odor hedonics on facial attractiveness: behavioural and fMRI measures, in: F. Signorelli (Ed.), Functional Brain Mapping and the Endeavor to Understand the Working Brain, InTech, 2007, pp. 209-225.

[10] J. Seubert, K.M. Gregory, J. Chamberland, et al., Odor valence linearly modulates attractiveness, but not age assessment, of invariant facial features in a memorybased rating task, PLoS One 9 (2014) e98347.

[11] S. Cook, N. Fallon, H. Wright, et al., Pleasant and unpleasant odors influence hedonic evaluations of human faces: an event-related potential study, Front. Hum. Neurosci. 9 (2015) 661.

[12] M. Bensafi, A. Pierson, C. Rouby, et al., Modulation of visual event-related poten tials by emotional olfactory stimuli, Clin. Neurophysiol. 32 (2002) 335-342.

[13] M. Smeets, G. Dijksterhuis, Smelly primes - when olfactory primes do or do not work, Front. Psychol. 5 (2014) 96.

[14] D.R. Herring, K.R. White, L.N. Jabeen, et al., On the automatic activation of attitudes: a quarter century of evaluative priming research, Psychol. Bull. 139 (2013) 1062-1089.

[15] J. De Houwer, D. Hermans, P. Eelen, Affective and identity priming with episodically associated stimuli, Cogn. Emot. 12 (1998) 145-169.

[16] D. Hermans, J. De Houwer, P. Eelen, A time course analysis of the affective priming effect, Cogn. Emot. 15 (2001) 143-165.

[17] A. Del Cul, S. Baillet, S. Dehaene, Brain dynamics underlying the nonlinear threshold for access to consciousness, PLoS Biol. 5 (2007) e260.

[18] T. Hummel, B. Sekinger, S.R. Wolf, et al., 'Sniffin' sticks': olfactory performance assessed by the combined testing of odor identification, odor discrimination and olfactory threshold, Chem. Senses 22 (1997) 39-52.

[19] N. Tottenham, J.W. Tanaka, A.C. Leon, et al., The NimStim set of facial expressions: judgments from untrained research participants, Psychiatry Res. 168 (2009) 242-249.

[20] D. Matsumoto, P. Ekman, Japanese and Caucasian Facial Expressions of Emotion (JACFEE) and Neutral Faces (JACNeuF) [Slides]: Dr. Paul Ekman, Department of Psychiatry, University of California, San Francisco, 1988.

[21] R.C. Gur, R. Sara, M. Hagendoorn, et al., A method for obtaining 3-dimensional facial expressions and its standardization for use in neurocognitive studies, J. Neurosci. Methods 115 (2002) 137-143.

[22] C.G. Kohler, T. Turner, W.B. Bilker, et al., Facial emotion recognition in schizophrenia: intensity effects and error pattern, Am. J. Psychiatry 160 (2003)
1768-1774.

[23] S. Cook, K. Kokmotou, V. Soto, et al., Pleasant and unpleasant odour-face combinations influence face and odour perception: an event-related potential study, Behav. Brain Res. 333 (2017) 304-313.

[24] J.M. Hopf, E. Vogel, G. Woodman, et al., Localizing visual discrimination processes in time and space, J. Neurophysiol. 88 (2002) 2088-2095.

[25] S. Bentin, T. Allison, A. Puce, et al., Electrophysiological studies of face perception in humans, J. Cogn. Neurosci. 8 (1996) 551-565.

[26] M. Kutas, K.D. Federmeier, Thirty years and counting: finding meaning in the N400 component of the event-related brain potential (ERP), Annu. Rev. Psychol. 62 (2011) 621-647.

[27] J.T. Cacioppo, S.L. Crites, G.G. Berntson, et al., If attitudes affect how stimuli are processed: should they not affect the event-related brain potential? Psychol. Sci. 4 (1993) 108-112.

[28] B.N. Cuthbert, H.T. Schupp, M.M. Bradley, et al., Brain potentials in affective picture processing: covariation with autonomic arousal and affective report, Biol. Psychol. 52 (2000) 95-111.

[29] G. Hajcak, J.P. Dunning, D. Foti, Neural response to emotional pictures is unaffected by concurrent task difficulty: an event-related potential study, Behav. Neurosci. 121 (2007) 1156-1162.

[30] G. Hajcak, J.S. Moser, R.F. Simons, Attending to affect: appraisal strategies modulate the electrocortical response to arousing pictures, Emotion 6 (2006) 517-522.

[31] A.K. Robinson, J. Reinhard, J.B. Mattingley, Olfaction modulates early neural responses to matching visual objects, J. Cogn. Neurosci. 27 (2014) 832-841.

[32] A. Leleu, O. Godard, N. Dollion, et al., Contextual odors modulate the visual processing of emotional facial expressions: an ERP study, Neuropsychologia 77 (2015) 366-379.

[33] E.R. Duval, J.S. Moser, J.D. Huppert, et al., What's in a face? The late positive potential reflects the level of facial affect expression, J. Psychophysiol. 27 (2013) 27-38.

[34] T. Dieguez-Risco, L. Aguado, J. Albert, et al., Faces in context: modulation of expression processing by situational information, Soc. Neurosci. 8 (2013) 601-620.

[35] N.K. Smith, J.T. Larsen, T.L. Chartrand, et al., Being bad isn't always good: affective context moderates the attention bias toward negative information, J. Pers. Soc. Psychol. 90 (2006) 210-220.

[36] N.K. Smith, J.T. Cacioppo, J.T. Larsen, et al., May I have your attention, please: electrocortical responses to positive and negative stimuli, Neuropsychologia 41 (2003) 171-183.

[37] T.A. Ito, J.T. Larsen, N.K. Smith, et al., Negative information weighs more heavily on the brain: the negativity bias in evaluative categorizations, J. Pers. Soc. Psychol. 75 (1998) 887-900.

[38] S. Boesveldt, J. Frasnelli, A.R. Gordon, et al., The fish is bad: negative food odors elicit faster and more accurate reactions than other odors, Biol. Psychol. 84 (2010) 313-317.

[39] B.E. Stein, T.R. Stanford, Multisensory integration: current issues from the perspective of the single neuron, Nat. Rev. Neurosci. 9 (2008) 255-266. 